\title{
A INFLUÊNCIA DA ALIMENTAÇÃO E ESTADO NUTRICIONAL DO BRASILEIRO SOB A INFLAMAÇÃO SUBCLÍNICA SISTÊMICA - UM PRATO CHEIO PARA O COVID-19
}

\author{
Maria Luisa Bellotto ${ }^{1}$
}

\begin{abstract}
Resumo
O conhecimento sobre hábitos alimentares; é relevante para compreensão da relação alimentação, obesidade e desenvolvimento de Doenças Crônicas Não Transmissíveis. O acúmulo de calorias em forma de gordura corporal, principalmente no tecido adiposo subcutâneo visceral, por consequência de uma alimentação hipercalórica e pobre em nutrientes, é responsável por gerar inflamação subclínica sistêmica sobrecarregando o sistema imunológico. A dieta desempenha importante papel na redução da inflamação. Em tempos de enfrentamento de uma pandemia de ordem infecciosa pelo novo corona vírus (COVID-19), este artigo tem o objetivo de discutir como a qualidade alimentar e o estado nutricional dos brasileiros interferem no enfrentamento desta infecção. Com base na literatura analisada, observamos que o hábito alimentar dos brasileiros tem favorecido o aumento de peso e a baixa ingestão de nutrientes, consequentemente aumentando a inflamação subclínica e diminuindo a capacidade de resposta imunológica a doenças inflamatórias. Sugerimos que as autoridades sanitárias se atentem para esta questão.
\end{abstract}

Palavras-chaves: Nutrição, Inflamação Subclínica, Obesidade, Covid-19

\section{Introdução}

A obesidade é decorrente do desequilíbrio positivo entre o consumo e o gasto energético (MANZUR; ALVEAR; ALAYÓN, 2010).

A estreita relação com o acúmulo de gordura corporal e o desenvolvimento de Doenças Crônicas Não Transmissíveis (DCNT). A compreensão biológica e social da obesidade, pode e deve auxiliar ações preventivas desta condição, considerada uma pandemia mundial que atinge países tanto em desenvolvimento como os desenvolvidos (SWINBURN, EGGER; RAZA, 1999; CROSSLEY, 2004). O acúmulo de calorias extras, principalmente no tecido adiposo subcutâneo visceral, somadas a uma alimentação pobre em nutrientes, e baixo gasto de energia por inatividade física podem desencadear respostas imunológicas que sobrecarregam o sistema de defesa deixando-os mais susceptíveis a infecções (FRANZ, 2014).

\footnotetext{
${ }^{1}$ Pesquisadora voluntária do Núcleo de Pesquisa em Movimento (NUPEM) da Universidade Metodista de Piracicaba (UNIMEP).
} 
De acordo com Verly Junior et al. (2013) num estudo realizado com mais de 1600 pessoas em São Paulo apontou que "pelo menos $80,0 \%$ da população consome abaixo do recomendado para: leite e derivados; frutas e sucos de frutas; e cereais, tubérculos e raízes; aproximadamente $60,0 \%$ para legumes e verduras; 30,0\% para feijões; e $8,0 \%$ para carnes e ovos. Adolescentes apresentaram a maior inadequação para legumes e verduras $(90,0 \%)$, e o estrato de maior renda foi associado à menor inadequação para óleos, gorduras e sementes oleaginosas (57,0\%)", segundo concluem os autores o consumo inadequado dos grupos de alimentos estão relacionados com aumento do risco de doenças crônicas.

Em tempos que enfrentamos outra pandemia de ordem infecciosa a nível mundial, o novo Corona Vírus (COVID - 19), acreditamos que o excesso de peso esteja intimamente relacionado com o deficiente prognóstico daqueles que venham se infectar com este vírus e sofrer com suas consequências (PENG, 2020 apud LAVIANO; KOVERECH; ZANETTI, 2020).

O objetivo deste artigo é discutir sobre a qualidade alimentar e o estado nutricional dos brasileiros, e apontar a sua vulnerabilidade para os possíveis riscos e complicações diante da exposição pelo COVID-19.

\section{Conhecimento dos hábitos alimentares e estado nutricional do brasileiro}

Os dados sobre o consumo alimentar da população adulta ainda são limitados por diversos motivos que impedem o real conhecimento de suas práticas (KIMOKOTI et al., 2012). Estudos realizados em alguns poucos países, como Espanha, Estados Unidos, Líbano e Austrália, o consumo alimentar de adultos foram analisados de modo sistematizado, utilizando para isso os inquéritos alimentares (Registro de Frequência Alimentar, Registro Alimentar ou Registro de 24 Horas) que permitiram a identificação com maior precisão os alimentos consumidos por grupos populacionais destas nacionalidades (MILLEN et al., 2005; NASREDDINE et al., 2006; MCNAUGHTON, et al., 2008).

No Brasil, os conhecimentos sobre o consumo alimentar são obtidos pelas extrapolações indiretas dos dados coletados pelas Pesquisa de Orçamentos Familiares (POFs), realizadas pelo Instituto Brasileiro de Geografia e Estatística (IBGE). A última edição da POF foi realizada em 2017-18, porém apenas dispomos nos dias de hoje os resultados preliminares. Este tipo de pesquisa oferece as tendências de consumo da população apenas para grupos dos alimentos. As POFs são realizadas com a finalidade econômica e busca estimar quanto o brasileiro emprega o seu dinheiro para comer e em quais grupos de alimentos. No entanto, 
este método limita a obtenção dos dados reais sobre o consumo alimentar das pessoas. Poucos estudos, como o de Mendonça et al. (2013), apresentam as preferências alimentares de adultos de uma cidade brasileira, como exemplo. Estudos de menor abrangência populacional e realizados em diversas faixas etárias e gênero também podem ser encontrados na literatura, porém eles foram realizados através de variadas metodologias de avaliação do consumo alimentar, dificultando a sistematização dos dados.

Segundo as informações dispostas pela POF anterior (2008-2009), 90\% da população come menos frutas, verduras e legumes que o recomendado, $68 \%$ consomem abaixo do índice ideal de fibras, $98 \%$ tem carência de vitaminas D e E no organismo, 86\% consomem mais gorduras saturadas que o necessário e 61\% abusam do açúcar (CONSELHO FEDERAL DE NUTRICIONISTAS, 2012). Desde que os dados atuais da POF 2017-2018 ainda não estão na sua totalidade disponíveis, podemos ao menos verificar que menos dinheiro está sendo empregado em frutas e verduras, importantes fontes de fibras e micronutrientes essenciais para o fortalecimento do sistema imunológico, quando comparados ao empregado no consumo de carnes, lácteos, bebidas industrializadas e bebidas alcoólicas.

Desde que os índices de obesidade mais do que dobraram no mundo desde a década de 80 , hoje $65 \%$ da população mundial vive em países onde o sobrepeso mata mais do que pessoas com baixo peso, apesar de que esta condição pode ser prevenida (WHO, 2011). No Brasil não é diferente, os dados da (GLOBAL NUTRITION REPORT, 2017) apontaram que entre os anos 2000 e 2016 o percentual de homens brasileiros com sobrepeso passaram de $47 \%$ para $55,6 \%$ e obesidade de $17,8 \%$ para $25,4 \%$, as mulheres neste mesmo período passaram de $44,8 \%$ para $55,4 \%$ o sobrepeso e de $11 \%$ para $18,5 \%$ a taxa de obesidade.

Infelizmente com a atual situação de pandemia por corona vírus, os períodos de distanciamento social está gerando uma situação particular de estresse e ansiedade, situações que podem gerar aumento do consumo de alimentos, principalmente os ricos em açúcares considerados "comfort food" (alimentos conforto) que consequentemente facilitam o ganho de peso desde que a atividade física possivelmente para muitos pode estar reduzda, devido ao fechamento de academias e espaços públicos para se exercitarem (FILGUEIRAS; STULTSKOLEHMAINEN, 2020).

Explicam Rodríguez-Martín e Meule (2015), que o desejo por consumir alimentos específicos inclui conceitos multidimensionais: o desejo por comer (emocional), a procurar por comida com ou sem razão (comportamental), pensamentos obsessivos por comida (cognitivo) e aumento da salivação (fisiológico). Segundo Muscogiuri et al. (2020), este fato 
ocorre mais em mulheres que homens, que costumam buscar por carboidratos desde que ajudam aumentar a produção de serotonina, substância que proporciona efeito positivo para o humor e o estresse.

\section{Obesidade e inflamação subclínica sistêmica}

O tecido adiposo possui diversas funções, cmo armazenamento de energia, regulação hormonal dos sistemas homeostáticos, termogênese e proteção de impacto contra as vísceras. No entanto, outra importante função do tecido adiposo é a função endócrina, ao secretar uma variedade de proteínas asintetizadas e liberadas pelos adipócitos, as chamadas adipocinas (BERG; SCHERER, 2005; BOOTH; MAGNUSON; FOSTER, 2014). As adipocinas são responsáveis pela: regulação, do apetite e balanço energético, imunidade, sensibilidade à insulina, angiogênese, inflamação e resposta de fase aguda, pressão sanguínea e metabolismo de lipídeos (FANTUZZI, 2005; MAFRA; FARAGE, 2006; GLEESON et al., 2011). Dentre as adipocinas mais conhecidas e estudadas no ambiente científico estão: leptina, adiponectina, resistina, fatores de crescimento, fatores de crescimento vascular, PCR, TNF- $\alpha$, IL-1, IL-6, IL-8, IL-10, IL-15, PGE2, inibidor de ativação do plasminogênio 1 (PAI-1) (TRAYHURN, WOOD, 2005; GREENBERG; OBIN, 2006; APOSTOLOPOULOS et al., 2016).

As alterações no balanço sistêmico e por consequência a instalação da inflamação subclínica sistêmica, influenciada pela presença de adipocinas inflamatórias liberadas por indivíduos obesos, podem resultar em um conjunto de doenças cardio-metabólicas, denominado Síndrome Metabólica (SM). Esta síndrome altera o metabolismo de tal forma a gerar DCNT, como dislipidemia, hipertensão arterial, doenças cardiovasculares e diabetes do tipo II entre outras (WILLERSON; RIDKER, 2004; FORD, 2005; BOOTH; MAGNUSON; FOSTER, 2014). Uma situação exacerbada com o avançar da idade (BRUUNSGAARD et al., 2004) e somadas a condição de estresse pode vir a alterar todo o estado inflamatório (NOWOTNY et al., 2010).

Maus hábitos de vida, podem somar aos efeitos da obesidade sob os processos de inflamação subclínica e o desenvolvimento de DCNT. O ato de fumar, como por exemplo, pode aumentar a quantidade de liberação de resistina, adipocina aumentada na condição da obesidade e altamente relacionada ao aumento na produção de glicose hepática, levando à resistência à insulina e a diabetes do tipo II (WALCHER et al., 2010; GURSOY, 2012). Portanto, um indvíduo obeso, com maus hábitos alimentares e de vida, é muito provável que 
tenha capacidade de defesa prejudicada, elevando-o a uma condição de maior susceptibilidade e debilidade imunológica.

\section{Hábitos alimentares e sua relação com os processos inflamatórios e o COVID-19}

A vida moderna leva as pessoas a realizarem hábitos alimentares inadequados, como comer fora de casa. Segundo dados da POF (2017-2018) mais de 30\% do dinheiro gasto porbrasileiros adultos são para realizar refeições fora de casa, esse número ainda é maior nas classes de rendas mais altas, chegando a superar mais de $50 \%$ do dinheiro total empregado a se alimentar. Segundo Batista Filho e Batista (2010), a realização das refeições fora das residências, pode atrair o indivíduo a consumir alimentos mais elaborados, mais saborosos e muitas vezes mais calóricos. O deslocamento do local de trabalho até o lar para almoçar pode ser complicado para os que vivem nas grandes cidades (distância, tempo, trânsito). Além disso, a preparação das refeições em casa é uma atividade que demanda tempo e dedicação (comprar os alimentos, prepará-los, cozinhá-los e limpar a sujeira). As mulheres nos dias de hoje também estão trabalhando fora para ajudarem no orçamento familiar, elas eram grandes aliadas para que as refeições pudessem ser elaboradas e/ou realizadas nos lares. Outros fatores favorecerem aolongo dos anos a transição do tradicional hábito alimentar do brasileiro: - a inexperiência de cozinhar, o aumento da oferta de produtos pré-preparado industrializados (muitas vezes de característica hipercalórica, ricos em sódio e conservantes e pobres em nutrientes e fibras), situações que favorecem o aumento do peso, agravado ainda mais pela falta ou redução de tempo para realização de atividade física (HERNANDEZ apud CANESQUI; GARCIA, 2005).

Tomar consciência desta situação e querer mudar é um grande avanço para melhorar a condição de saúde. Acreditamos que este ponto é de extrema importância a ser abordado por políticas públicas, que devem visar a educação nutricional dos brasileiros, ao encorajar que as comidas sejam trazidas de casa ao trabalho ou a adequação das preparações oferecidas pelos estabelecimentos. (OLIVEIRA; ABRANCHES; LANA, 2020).

O hábito alimentar típico do brasileiro se caracteriza por baixa ingestão de fibras alimentares (presentes em alimentos como leguminosas, verduras e frutas), elevado consumo de gorduras saturadas e trans e, açúcares simples (encontrados em produtos industrializados como bolachas, refrigerantes, sucos, salgadinhos, salgados, alimentos industrializados de rápido preparo, bebidas alcoólicas, pizza, sanduíches, chocolates), aliados à redução do gasto 
energético geram um balanço positivo que contribui para o ganho de peso caracterizado pelo acúmulo de gordura corporal, (OLIVEIRA, 2007; LOTTENBERG, 2009; BRASIL, 2013).

Em relação as gorduras, os ácidos graxos do tipo saturado (presentes em carnes e produtos derivados animais, gorduras hidrogenadas e óleo de côco e dendê) e os ácidos graxos trans. (gorduras parcialmente hidrogenadas, óleos refinados, gordura animal: carne, leite e derivados) são grandes responsáveis por induzirem uma resposta inflamatória crônica no organismo (MARTIN; MATSHUSHITA; SOUZA, 2004). Segundo descreve Murumalla (2012) dietas ricas em gorduras levam a hipertrofia e inflamação dos adipócitos que liberam citocinas pro inflamatórias e ácidos graxos livres (AGL) via lipólise. Os AGL liberados no plasma através de lipoproteínas, especialmente os ácidos graxos saturados, desencadeiam a inflamação no tecido adiposo, gerando condições para a ocorrência de doenças cardiovasculares como a arteriosclerose e prejudicando a via de sinalização da insulina no tecido muscular (BULLÓ et al., 2007), um tema que vem sendo discutido nos dias de hoje incluindo a interferência da composição de dietas como um todo e o tipo de flora intestinal como influenciadores do grau da resposta inflamatória (FRITSCHE, 2015). O consumo excessivo deste tipo de gordura induz o estado lipotóxico, ativando o Sistema imune inato através de toll-like receptor 4 expressos por macrófagos, células dendriticas e neutrófilos e inibindo o sistema imune adaptativo. Essa condição aumenta o estresse oxidativo e a imunodepressão, amplificando o risco de patologias geradas pela infecção do COVID-19 (BUTLER; BARRIENTOS, 2020).

Ao contrário, os ácidos graxos do tipo poli saturados da série $\varpi-3$, como o ácido eicosapentaenoico (EPA) e ácido docosaexaenoico (DHA), desempenham papel protetor (SIMOPOULOS, 2002). Presentes em peixes, óleos de peixe, óleo de canola, azeite e oleaginosas, apresentam ação anti-inflamatória, demonstrados em diversos tipos de células e modelos experimentais tanto animais e humanos, que proporcionam proteção para a saúde ao diminuírem a secreção das citocinas pró-inflamatórias (MURUMALLA, 2012). O impoetante é mantwr o equilíbrio do conumo de todos os tipos de gordura, através de uma alimentação balanceada. Informação que deve ser transmitida por processos educativos, valorizando e ao mesmo tempo questionando os aspectos culturais e a riqueza dos alimentos cultivados e produzidos pelo nosso rico país.

Outras substâncias de baixo peso molecular que possuem potente atividade biológica são os compostos bioativos presentes em frutas e verduras. Segundo Horst e Lajolo (2011) os compostos bioativos são indispensáveis por não serem sintetizados pelo organismo humano, e 
apresentam ação protetora na saúde humana quando presentes na dieta em quantidades significativas. Dentre as atividades biológicas por eles realizadas estão: atividade antioxidante, modulação de enzimas de destoxificação, estimulação do sistema imune, redução da agregação plaquetária, modulação do metabolismo hormonal, redução da pressão sanguínea, e atividade antibacteriana e antiviral, sinalizados pelos marcadores inflamatórios acima relacionados.

\begin{abstract}
"Em um novo paradigma, a ingestão insuficiente de compostos bioativos provenientes de vegetais constitui importante componente de risco das DCNT, contribuindo na mesma magnitude do consumo excessivo de energia e de gorduras totais e saturadas na dieta. Isso indica que os compostos bioativos, da mesma forma que os demais nutrientes, são essenciais para que se atinja a carga completa (geneticamente determinada) de longevidade. Segundo esse novo paradigma, as DCNT seriam doenças relacionadas também à deficiência de substâncias "essenciais para a longevidade (BASTOS; ROGERO; ÁREAS, 2009).
\end{abstract}

E para finalizar, estimular o consumo regular de fibras, do tipo solúvel e insolúvel, elas podem amenizar problemas de ordem cardiovascular ao reduzir as concentrações séricas da LDL colesterol, além de contribuir para o controle do peso corporal, tolerância à glicose e incidência de Diabetes tipo 2 e redução da inflamação crônica (RIQUE; SOARES; MEIRELLES, 2002; SATIJA; HU, 2012). Encontradas em frutas, legumes, cereais integrais, grãos e oleaginosas. Dito isso, não nos resta dúvidas sobre a influência que o excesso de peso gera no sistema de defesa e como isso implica na capacidade de respostas inflamatórias, seja de ordem infecciosa ou inflamatória. Demonstrando, portanto, a importância do controle de peso corporal e da realização de bons hábitos alimentares para uma melhor capacidade de resposta imunológica em combate, neste momento, a infecção pelo COVID-19.

\title{
Alimentação e tempos de pandemia
}

O COVID-19, causado pelo novo corona vírus, SARS-CoV-2, alcançou o estado de pandemia a nível mundial em 2020. Este vírus altamente contagioso pode afetar o metabolismo de maneira sistêmica de forma eminente, prejudicando e sobrecarregando o sistema de defesa humano a ponto de levar a uma taxa de letalidade muito alta, principalmente entre pessoas de maior idade e com a presença de comorbidades (SARZIPUTTINI et al., 2020).

Quanto a quarentena, muitos relatam um aumento do estresse gerado pelo isolamento social, insegurança no trabalho, redução ou perda total da renda, instabilidade política e 
sanitária, violência doméstica e outros problemas comumente relatados pela mídia brasileira nos dias de hoje (LIMA et al., 2020). Este estresse pode gerar também distúrbios no sono e que por sua vez gera distúrbios no comportamento alimentar, como a ansiedade (NAJA; HAMADEH, 2020).

Contra este mal, Muscogiuri et al. (2020) recomendam o consumo de alimentos que promovam a síntese de serotonina e melanina, principalmente na refeição do jantar. Dentre estes alimentos se destacam o consumo de raízes, folhas, frutas, sementes e cereais integrais. Os autores ainda destacam o leite e derivados que contém triptofano, um precursor de serotonina e melatonina, que ajudam a induzir o sono além de aumentar a atividade celular de natural killers e assim reduzir o risco de infecção respiratória. Além disso, o triptofano está envolvido no sistema regulatório da saciedade via liberação da serotonina que ajuda a reduzir o desejo por carboidratos e gorduras e inibe neuropeptídios $Y$, peptídeos orexígenos hipotalâmicos mais poderosos. Os autores ainda lembram que o aumento do consumo de alimentos ricos em carboidratos, acompanham a redução dos alimentos fontes de micronutrientes, um comportamento comum entre pessoas obesas, que se somado a inatividade física eleva o indivíduo a uma condição mais susceptível de infecção ao vírus COVID-19, tal como relatamos anteriormente.

Naja e Hamadeh (2020), sugerem que uma série de recomendações podem ser realizadas a nível individual, coletivo e até nacional. A nível individual, os autores recomendam sustentar o funcionamento do Sistema imunológico, ao incluir refeições equilibradas, evitar "beliscos" irregulares, ingerir alimentos ricos em vitamina A, C, E, B6, B12, zinco e ferro, como: frutas cítricas, vegetais verdes folhosos escuros, oleaginosas, lácteos. Além de manter uma rotina saudável, com a prática de atividade física, regularidade do sono, meditação, evitar fumar, consumir álcool e drogas. São recomendações que seguem as mesmas orientações proporcionadas pela FAO (2020) para este momento de pandemia, e que ainda sugere o consumo de cereais integrais, sementes, azeite de oliva e se evite alimentos ricos em gordura, açúcares e sal e reforça o consumo de água. A agência internacional sugere que as pessoas devem se abster de passar informações duvidosas sobre práticas alimentares e o COVID-19.

\section{Conclusão}

$\mathrm{O}$ acúmulo de gordura principalmente na região abdominal, caracterizando o sobrepeso e obesidade, reflexo de uma má alimentação e consumo de alimentos de alta 
densidade calórica e baixo em nutrientes, estão intimamente relacionados ao desencadeamento de processos inflamatórios e consequentemente o desenvolvimento de DCNT, o que fragiliza o sistema de defesa e torna o indivíduo mais susceptíveis a infecções. Em contrapartida, estudos indicam que a alimentação adequada desempenha o importante papel na redução do risco de doenças e fortalecimento do sistema imunológico. Os hábitos alimentares dos brasileiros apresentam falhas que favorecem o aumento do peso e a baixa ingestão de nutrientes importantes para o sistema de defesa. Consequentemente, essa condição não favorece os indivíduos que possam vir a se contaminar pelo Covid-19. Com base ao exposto anteriormente, não resta dúvidas que as autoridades sanitárias, da esfera Federal a Municipal, devam atentar-se para esta problemática, promovendo políticas e ações informativas.

\section{Referências}

APOSTOLOPOULOS, V. et al. The complex immunological and inflammatory network of adipose tissue in obesity. Mol. Nutr. Food Res., Weinheim, v. 60, n. 1, p. 43-57, 2016.

BASTOS, D. H. M.; ROGERO, M. M.; AREAAS, J. A. G. Mecanismos de ação de compostos bioativos dos alimentos no contexto de processos inflamatórios relacionados à obesidade.

Arq. Bras. Endocrinol. Metab. São Paulo, v. 53, n. 5, p. 646-656, 2009.

BATISTA FILHO, M.; BATISTA, L. V. Transição alimentar/ nutricional ou mutação antropológica? Cienc. Cult., São Paulo, v. 62, n. 4, p. 26-30, 2010.

BERG, A. H.; SCHERER, E. P. Adipose tissue, inflamation, and cardiovascular disease.

Circ. Res., Baltimore, v. 96, p. 939-949, 2005.

BOOTH, A.; MAGNUSON, A.; FOSTER, M. Detrimental and protective fat: body fat distribution and its relation to metabolic disease. Horm. Mol. Biol. Clin. Investig., Berlin, v. 17, n. 1, p. 13-27, 2014.

BRASIL. Ministério da Saúde. Portal da Saúde. Vigilância de doenças crônicas não transmissíveis. Brasília: Ministério da Saúde, 2013. Disponível em:

$\mathrm{http}: / /$ portal.saude.gov.br/portal/saude/profissional/visualizar_texto.cfm?idtxt=31877. Acesso em: 29 jan. 2019.

BRUUNSGAARD, H. et al. The tumor necrosis factor Alpha $-308 \mathrm{G}>$ a polymorphism is associated with dementia in the oldest old. J. Am. Geriatr. Soc., New York, v. 52, n. 8, p. 1361-1366, 2004.

BULLÓ, M. et al. Inflammation, obesity and comorbidities: the role of diet. Public Health Nutr., Wallingford, v. 10, n. 10A, p. 1164-1172, 2007.

BUTLER, M. J.; BARRIENTOS, R. M. The impact of nutrition on COVID-19 susceptibility and long-term consequences. Brain Behav. Immun. v. 87, p. 53-54, 2020. Disponível em: https:// https://doi.org/10.1016/j.bbi.2020.04.040. Acesso em: 18 abr. 2020 
CANESQUI, A. M.; GARCIA R. W. D. (org.). Antropologia e nutrição: um diálogo possível. Rio de Janeiro: Editora Fiocruz, 2005.

CONSELHO FEDERAL DE NUTRICIONISTAS. Alimentação fora do lar: campanha destaca importância da categoria para os hábitos alimentares. Rev. CFN, Brasília, v. 38, p. 45-53, 2012.

CROSSLEY, N. Fat is a sociological issue: obesity rates in late modern, 'body-conscious' societies. Soc. Theory Health, Basingstoke, v. 2, p. 222-253, 2004.

DUARTE, C. P.; SANTOS, C. L.; GONÇALVEZ, A. K. A concepção de pessoas de meia idade sobre saúde, envelhecimento e atividade física como motivação para comportamentos ativos. Rev. Bras. Cienc. Esporte, Campinas, v. 23, n. 3, p. 5-48, 2002.

FANTUZZI, G. Adipose tissue, adipokines, and inflammation. J. Allergy Clin. Immunol. St Louis, v. 115, p. 911-919, 2005.

FILGUEIRAS, A.; STULGTS-KOLEHMAINEN, M. Factors linked to changes in mental health outcomes among Brazilians in quarantine dut to COVID-19. MedRxiv, Cold Spring Harbor, 2020. Disponível em: https://doi.org/10.1101/2020.05.12.20099374. Acesso em: 26 ago. 2020.

FOOD AND AGRICULTURE ORGANIZATION. Maintaining a healthy diet during the COVID-19 pandemic. Rome: FAO, 2020.

FORD, E. S. Risks for all-cause mortality, cardiovascular disease, and diabetes associated with the metabolic syndrome: a summary of the evidence. Diabetes Care, New York, v. 28, p. 1769-1778, 2005.

FRANZ, M. Nutrition, inflammation and desease. Todays's Dietitian, Spring City, v. 16, n. 2, p. 44, 2014.

FRITSCHE, K. L. The science of fatty acids and inflammation. Adv. Nutr., Bethesda, v. 6, n. 3, p. 293S-301S, 2015.

GLEESSON, M. et al. The anti-inflammatory effects of exercise: mechanisms and implications for the prevention and treatment of disease. Nat. Rev. Immunol. London, v. 11, n. 9, p. 607-615, 2011.

GLOBAL NUTRITION REPORT. Adult nutrition status. [S. 1.]: Global Nutrition Report, 2017. Disponível em: https://globalnutritionreport.org/resources/nutrition-profiles/latinamerica-and-caribbean/south-america/brazil/\#profile. Acesso em: 12 maio 2020.

GREENBERG, A. S.; OBIN, M. Obesity and the role of adipose tissue in inflammation and metabolism. Am. J. Clin. Nutr., Bethesda, v. 83, n. 2, p. 461S-465S, 2006.

GURSOY, G. et al. The relation of obesity with serum resistin levels in smoker and nonsmokers. J. Res. Med. Sci., Isfahan, v. 17, n. 2, p. 119-122, 2012.

HORST, M. A.; LAJOLO, F. M. Biodisponibilidade de compostos bioativos de alimentos. Belo Horizonte: Nutrição Clínica Estética, 2011.

IBGE. Pesquisa de Orçamentos familiares - POF: POF 2017-2018: avaliação nutricional da disponibilidade domiciliar de alimentos no Brasil. Rio de Janeiro: IBGE, 2019. Disponível 
em: https://www.ibge.gov.br/estatisticas/sociais/educacao/24786-pesquisa-de-orcamentosfamiliares-2.html?edicao=27139\&t=resultados. Acesso em: 13 maio 2020.

IBGE. Pesquisa de Orçamentos Familiares -POF: POF 2008-2009: antropometria e estado nutricional de crianças, adolescentes e adultos no Brasil. Rio de Janeiro: IBGE, 2010.

Disponível em:

http://www.ibge.gov.br/home/estatistica/populacao/condicaodevida/pof/2008_2009_encaa/po f_20082009_encaa.pdf. Acesso em: 10 mar. 2019.

INSTITUTE OF MEDICINE. Dietary reference intakes. Tables of DRI values. Washington (DC): National Academy Press, 2011. Disponível em:

http://iom.edu/Activities/Nutrition/SummaryDRIs/DRI-Tables.aspx. Acesso em: 12 abr. 2014.

KIMOKOTI, R. W. et al. Dietary patterns of women are associated with incident abdominal obesity but not metabolic syndrome. J. Nutr., Rockville, v. 142, n. 9, p. 1720-1727, 2012.

LAVIANO, A.; KOVERECH, A.; ZANETTI, M. Nutrition support in the time of SARSCoV-2 (COVID-19). Nutrition, Tarrytown, v. 74, p. 110834, 2020.

LIMA, S. O. et al. Impactos no comportamento e na saúde mental de grupos vulneráveis em época de enfrentamento da infecção COVID-19: revisão narrativa. Rev. Eletrôn. Acervo Saúde, Brasil, n. 46, p. e4006, 2020. Disponível em:

https://doi.org/10.25248/reas.e4006.2020. Acesso em: 26 ago. 2020.

LOTTENBERG, A. M. P. Importância da gordura alimentar na prevenção e no controle de distúrbios metabólicos e da doença cardiovascular. Arq. Bras. Endocrinol. Metab., São Paulo, v. 53, n. 5, p. 595-607, 2009.

MAFRA, D.; FARAGE, N. E. O papel do tecido adiposo na doença renal crônica. J. Bras. Nefrol., Rio de Janeiro, v. 28, n. 2, p. 108-113, 2006.

MANZUR, F.; ALVEAR, C.; ALAYÓN, A. N. Adipocitos, obesidad visceral, inflamación y enfermedad cardiovascular. Rev. Colomb. Cardiol., Bogota, v. 17, n. 5, p. 207-213, 2010.

MARTIN, C. A.; MATSHUSHITA, M.; SOUZA, N. E. Ácidos graxos trans: implicações nutricionais e fontes na dieta. Rev. Nutr., Campinas, v. 17, n. 3, p. p. 351-359, 2004.

MCNAUGHTON, S. A. et al. An index of diet and eating patterns is a valid measure of diet quality in an australian population. J. Nutr., Rockville, v. 138, n. 1, p. 86-93, 2008.

MENDONÇA, S. N. et al. Food preferences of middle aged and elderly subjects in a Brazilian city. J. Nutr. Health Aging, Paris, v. 17, n. 2, p. 130-135, 2013.

MILLEN, B. E. et al. Unique dietary patterns and chronic disease risk profiles of adult men: the Framingham nutrition studies. J. Am. Diet. Assoc., Chicago, v. 105, n. 11, p. 1723-1734, 2005 .

MURUMALLA, R. K. et al. Fatty acids do not pay the toll: effect of SFA and PUFA on human adipose tissue and mature adipocytes inflammation. Lipids Health Dis., London, v. 11, p. 175, 2012.

MUSCOGIURI, G. et al. Nutritional recommendations for CoVID-19 quarantine. Eur. J. Clin. Nutr., London, v. 74, p. 850-885, 2020. 
NAJA, F.; HAMADEH, R. Nutrition amid the COVID-19 pandemic: a multi-level framework for action. Eur. J. Clin. Nutr., London, v. 74, n. 8, p. 1117-1121, 2020.

NASREDDINE, L. et al. Food consumption patterns in an adult urban population in Beirut, Lebanon. Public Health Nutr., Wallingford, v. 9, n. 2, p. 194-203, 2006.

NOWOTNY, B. et al. Effects of acute psychological stress on glucose metabolism and subclinical inflammation in patients with post-traumatic stress disorder. Horm. Metab. Res., Stuttgart, v. 42, n. 10, p. 746-753, 2010.

OLIVEIRA, A. Alimentação e inflamação. Rev. Factores Risco, Lisboa, v. 5, p. 71, 2007.

OLIVEIRA, T. C.; ABRANCHES, M. V.; LANA, R. M. (In) Segurança alimentar no contexto da pandemia por SARS-CoV-2. Cad. Saúde Pública, Rio de Janeiro, v. 36, n. 4, p. e00055220, 2020 .

RIQUE, A. B. R.; SOARES, E. A. E.; MEIRELLES, C. M. Nutrição e exercício na prevenção e controle das doenças cardiovasculares. Rev. Bras. Med. Esporte, Niterói, v. 8, n. 6, p. 244$254,2002$.

RODRÍGUEZ-MARTÍN, B. C.; MEULE, A. Food craving: new contributions on its assessment, moderators, and consequences. Front. Psychol., Pully, v. 6, p. 21, 2015.

SARZI-PUTTINI, P et al. COVID-19, cytokines and immunosuppression: what can we learn from severe acute resiratory syndrome? Clin. Exp. Rheumatol., Pisa, v. 38, n. 2, p. 337-342, 2020 .

SATIJA, A.; HU, F. B. Cardiovascular benefits of dietary fiber. Curr. Atheroscler. Rep., Philadelphia, v. 14, n. 6, p. 505-514, 2012.

SIMOPOULOS, A. P. Omega-3 fatty acids in inflammation and autoimmune diseases. J. Am. Coll. Nutr., New York, v. 21, n. 6, p. 495-505, 2002.

SWINBURN, B.; EGGER, G.; RAZA, F. Dissecting obesogenic environments: the development and application of a framework for identifying and prioritizing environmental interventions for obesity. Prev. Med., New York, v. 29, p. 63-570, 1999.

TRAYHURN, P.; WOOD, I. S. Signalling role of adipose tissue: adipokines and inflammation in obesity. Biochem. Soc. Trans., London, v. 33, n. 5, p. 1078-1081, 2005.

VERLY JUNIOR, et al. Adesão ao guia alimentar para população brasileira. Rev. Saúde Pública, São Paulo, v. 47, n. 6, p. 1021-1027, 2013.

WALCHER, D. et al. Resistin: a new identified chemokine for human CD4-positive lymphocytes. Cardiovasc. Res., Oxford, v. 85, p. 167-174, 2010.

WILLERSON, J. T.; RIDKER, P. M. Inflammation as a cardiovascular risk factor. Circulation, Hagerstown, v. 109, p. 2-10, 2004.

WORLD HEALTH ORGANIZATION. Obesity and overweight. Geneva: WHO, 2011. (Fact sheet n. 311). Disponível em: http://www.who.int/mediacentre/factsheets/fs311/en/> Acesso em: 2 fev. 2019. 


\title{
THE INFLUENCE OF FOOD AND NUTRITIONAL STATUS OF BRAZILIANS ON THE RISK OF SUBCLINICAL SYSTEMIC INFLAMMATION - A FULL DISH FOR COVID-19
}

\begin{abstract}
Knowledge about eating habits is relevant for understanding the relations between diet, obesity and development of Chronic Non-Communicable Diseases. The storage of calories as body fat, mainly in the visceral subcutaneous adipose tissue, as consequence of hypercaloric diet poor in nutrients, is responsible for increasing sublinic inflammation and overloading the immune system. Diet plays an important role in reducing inflammation. In time of infectious pandemia, of the new corona virus (COVID-19), the food quality and nutritional status of Brazilians may interfere in fighting this infection. Based on the literature, this article aims to discuss the Brazilians eating habbits and nutritional status. The data shows that Brazilian are gainning weight and tend to a diet poor in nutrient intake. Consequently this scenario favors the subclinical inflammation and the weakness of the immune response. We suggest that Brazilian Health Authorities pay attention to this issue.
\end{abstract}

Keywords: Nutrition, Subclinical Inflammation, Obesity, Covid-19

\section{LA INFLUENCIA DE LA ALIMENTACIÓN Y DEL ESTADO NUTRICIONAL DE BRASILENOS SOB LA INFLAMACIÓN SUBCLÍNICA SISTEMICA - UN PLATO LLENO PARA EL COVID-19}

\begin{abstract}
Resumen
El conocimiento sobre los hábitos alimenticios es relevante para comprender la relación entre la dieta, la obesidad y el desarrollo de enfermedades crónicas no transmisibles. El acumulo de calorías en forma de grasa corporal, principalmente en el tejido adiposo subcutáneo visceral, como consecuencia de una dieta hipercalórica y pobre en nutrientes, es responsable de generar inflamación sublínica sistémica que sobrecarga el sistema inmune. La dieta juega un papel importante en la reducción de la inflamación. En tiempos de enfrentamento de una pandemia infecciosa por el nuevo virus corona (COVID-19), este artículo tiene como objetivo discutir cómo la calidad de los alimentos y el estado nutricional de los brasileños interfieren para hacer frente a esta infección. Con base en la literatura analizada, observamos que los hábitos alimenticios de los brasileños han favorecido el aumento de peso y la baja ingesta de nutrientes, lo que aumenta la inflamación subclínica y disminuye la respuesta inmune a las enfermedades inflamatorias. Sugerimos que las autoridades de salud presten atención a este problema.
\end{abstract}

Palabras clave: Nutrición, Inflamación Subclínica, Obesidad, Covid-19 


\section{Agradecimentos}

Agradeço a oportunidade incentivada pela professora Dra. Rute Estanislava Tolocka (NUPEM/UNIMEP), em escrever este artigo. E as professoras Claudia Regina Cavagliere e Mara Patrícia Chacon-Mikahil (FISEX/INICAMP), por me permitirem desenvolver os estudos de nutrição voltada a inflamação subclínica sistêmica, como pesquisadora colaborada do laboratório de fisiologia do exercício da Faculdade de Educação Física da Unicamp, oportunidade que gerou as condições para a apresentação deste artigo. 